\title{
A Radioactive-free Kinase Inhibitor Discovery Assay Against the Trypanosoma brucei Glycogen Synthase Kinase-3 short (TbGSK-3s) \\ Antonia Efstathiou ${ }^{1, \$, *}$ and Despina Smirlis ${ }^{1}$
}

\begin{abstract}
${ }^{1}$ Molecular Parasitology Lab, Dpt of Microbiology, 127 Bas. Sofias Ave., Hellenic Pasteur Institute, 11521

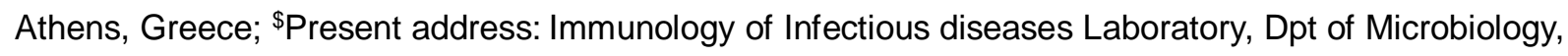
127 Bas. Sofias Ave., Hellenic Pasteur Institute, 11521 Athens, Greece
\end{abstract}

*For correspondence: toniaef@pasteur.gr or toniaefstathiou@gmail.com

[Abstract] The identification of small molecules possessing inhibitory activity in vitro, against a given target kinase, is the first step in the drug discovery process. Herein, we describe a non radioactive protocol using luciferase-based ATP assay for the identification of inhibitors for the short isoform of the Trypanosoma bruceis Glycogen Synthase Kinase-3 (TbGSK-3s). TbGSK-3s represents a potential drug target as it is essential for parasite survival. Small molecules used in our study are indirubin analogues possessing substitutions in different positions in the bis-indole backbone. Presently, the standard laboratory practice for the kinase assays is the incorporation of radiolabeled phosphate from [gamma${ }^{32}$ PJATP as the efforts for developing non-radioactive assays (ELISA-based assays, fluorescence quenching assays, etc.) exhibit limitations such as lack in sensitivity or limitations for broad applications. This protocol can be a useful starting point for lead discovery, as it surpasses the drawbacks of radioactive kinase assays and it allows for relatively sensitive measurements of kinase inhibition for TbGSK-3s.

Keywords: Kinase assay, Non-radioactive assay, Trypanosoma brucei, TbGSK-3s, Lead discovery, Inhibitors, Indirubins

[Background] Kinases are enzymes that play a crucial role in biological processes including differentiation, cell proliferation and apoptosis, by putting in motion signaling pathways upon catalyzing the transfer of the Y-phosphate from ATP to substrate (Jia et al., 2008; Efstathiou et al., 2019). Deregulation of kinases can frequently lead to a variety of diseases (Ways and Sheetz, 2000; Cohen and Goedert, 2004; Mazitschek and Giannis, 2004; Resnick and Fennell, 2004) and therefore, they are considered one of the largest classes of drug targets (Cohen 1999; Manning et al., 2002). The first step of the lead kinase inhibitor discovery is the establishment of an in vitro kinase assay. The radioactive assays are the standard laboratory practice due to their high sensitivity (Jia et al., 2008; Lilienthal et al., 2010). However, drawbacks of kinase-based radioactivity assays include the need of special handling and the restriction in flexibility because of the short half-life of ${ }^{32} \mathrm{P}$. To resolve these limitations, new nonradioactive technologies have been created that are based on fluorescence or luminescence (Jia et al., 2008). Herein, we describe a non radioactive protocol using luciferase-based ATP assay, for the identification of inhibitors for the short isoform of the Trypanosoma bruceis Glycogen Synthase Kinase3 (TbGSK-3s). In the bloodstream form of T. brucei, TbGSK-3s is essential for survival (Ojo et al., 2008) 
and therefore it is a molecular target for the discovery of new anti-trypanosomal agents (Ojo et al., 2008; Oduor et al., 2011; Woodland et al., 2013; Urich et al., 2014; Swinney et al., 2016). Mammalian GSK-3 has been related to a wide range of diseases and thus small molecular weight GSK-3 inhibitors has been developed (Woodland et al., 2013; Gaboriaud-Kola et al., 2015; Masch and Kunick, 2015). Amongst GSK-3 inhibitors, there are the indirubins, a family of natural bis-indole derivatives (Hoessel et al., 1999, Polychronopoulos et al., 2004, Vougogiannopoulou et al., 2008, Myrianthopoulos et al., 2013). In this protocol, a luminescent kinase assay based on the Kinase-Glo ${ }^{\circledR}$ reagent of Promega, is described. This method is straightforward, radioactive-free, fast and it doesn't lack sensitivity. While the protocol described below is specific for the recombinant TbGSK-3s expressed in baculovirus system as described before (Efstathiou et al., 2019), it can be applicable to any kinase with the appropriate alterations for the specific kinase (substrate, ATP concentration, buffer, etc.).
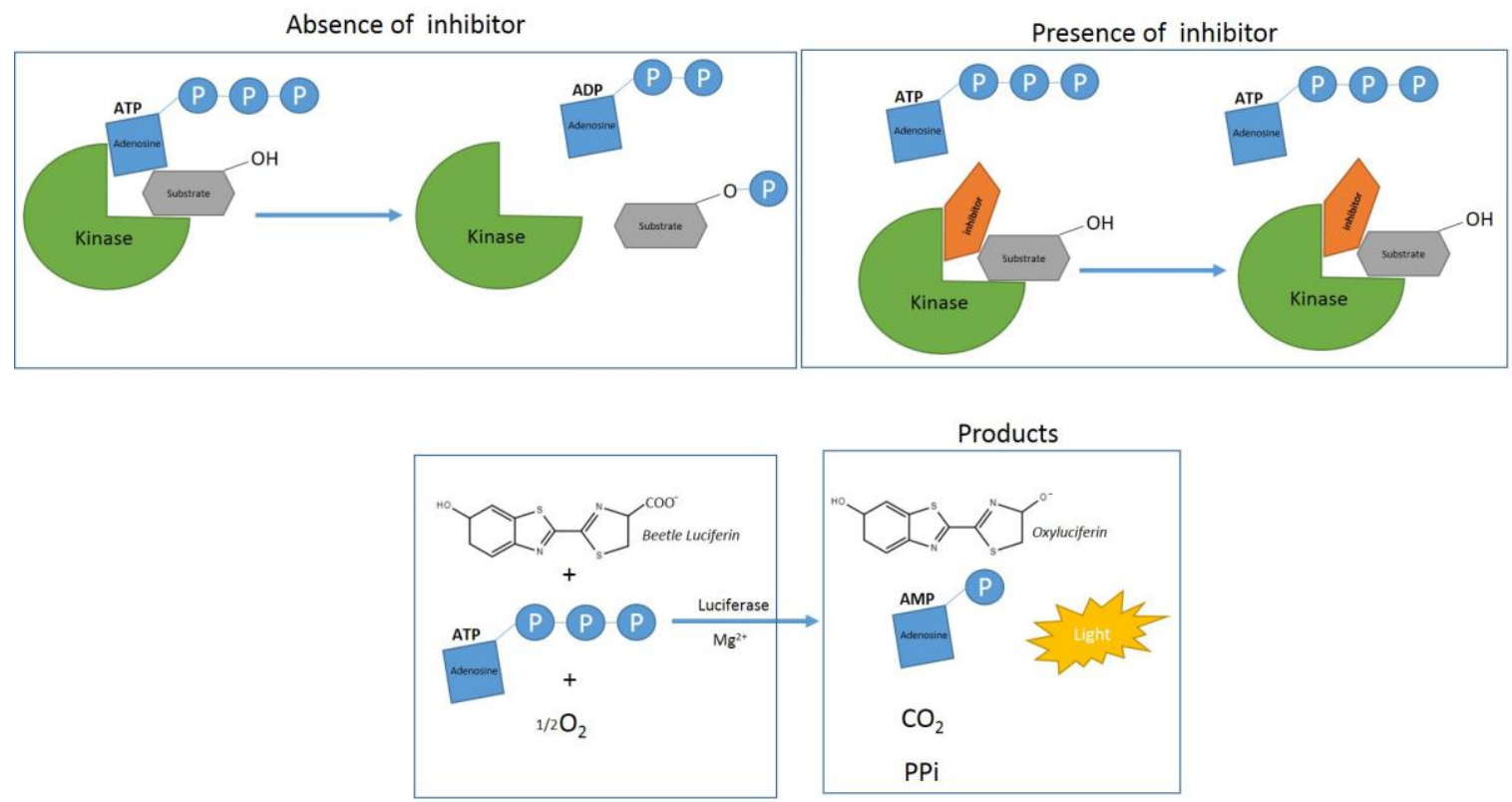

Figure 1. Scheme for the luminescent kinase assay based on the Kinase-Glo ${ }^{\circledR}$ reagent. The kinase reaction is conducted under the appropriate conditions with or without inhibitors. The remaining ATP at the time that the reagent is added, is used as a substrate by the Kinase-Glo ${ }^{\circledR}$ Luciferase to catalyze the mono-oxygenation of luciferin. The luciferase reaction produces one photon of light per turnover. Luminescence is inversely related to kinase activity (Promega).

\section{Materials and Reagents}

\section{Materials}

1. Pipettes tips: $0.5-10 \mu \mathrm{l}, 10-200 \mu \mathrm{l}, 200-1,000 \mu \mathrm{l}$ (Greiner Bio-One, catalog numbers: 771291 , 739290, 740290)

2. Eppendorf tubes (Greiner Bio-One, catalog number: 616201)

3. Amicon ${ }^{\circledR}$ Ultra-4 centrifugal filters 


\section{Reagents}

1. GSK-3 peptide substrate YRRAAVPPSPSLSRHSSPHQ(pS)EDEEE (HQ), 1 mg (Biaffin GmbH \& Co KG, proteinkinase.de, catalog number: PEP-GSK-001, storage temperature: $-20^{\circ} \mathrm{C}$ )

2. Kinase-Glo ${ }^{\circledast}$ Luminescent Kinase Assay, $10 \mathrm{ml}$ (Promega, catalog number: V6711, storage temperature: $-20^{\circ} \mathrm{C}$ )

3. Kinase Glo ${ }^{\circledast}$ Substrate, 1 vial (lyophilized) (Promega, catalog number: V378A)

4. Kinase $\mathrm{Glo}^{\circledR}$ Buffer, $10 \mathrm{ml}$ (Promega, catalog number: V379A)

5. Adenosine $5^{\prime}$-triphosphate (ATP) disodium salt hydrate, $1 \mathrm{mg}$ (Sigma-Aldrich, catalog number: FLAAS, storage temperature: $-20^{\circ} \mathrm{C}$ )

6. TbGSK-3s expressed in a baculovirus expression system as previously described (Efstathiou et al., 2019) (storage temperature: immediate usage after expression or glycerol stock at $-80^{\circ} \mathrm{C}$ ) Notes:

a. TbGSK-3s is not available commercially. In order to use it, it must be expressed in the laboratory following the protocols described in bibliography (Efstathiou et al., 2019). Briefly, as mentioned in Efstathiou et al., 2019, the pTriEx-1.1-TbGSK3s plasmid was cotranfected with the BaculoGold DNA into Spodoptera frugiperda (Sf9) insect cells and upon production of the TbGSK3s, the kinase was purified on $\mathrm{Ni}^{2+}$-nitrilotriacetate (Ni-NTA) resin according to the manufacturer's instructions (Qiagen).

b. Kinase fractions should be used immediately to ensure maximum activity. If they are stored as glycerol stocks at $-80^{\circ} \mathrm{C}$, they should be used up to 3-5 days upon isolation to avoid complete loss of the kinase activity.

7. 32 indirubin analogs that were synthesized as previously described (Meijer et al., 2003; Polychronopoulos et al., 2004; Ferandin et al., 2006; Vougogiannopoulou et al., 2008), (storage temperature: $4^{\circ} \mathrm{C}$, away from sunlight)

8. MOPS (3-(N-morpholino)propanesulfonic acid) (Applichem, catalog number: A2947, storage temperature: RT)

9. $\mathrm{MgCl}_{2}$ (Magnesium chloride hexahydrate) (Applichem, catalog number: A1036, storage temperature: RT)

10. EGTA (Ethylene glycol-bis(2-aminoethylether)-N,N,N',N'-tetraacetic acid) (Sigma-Aldrich, catalog number: E4378, storage temperature: RT)

11. Pierce ${ }^{\text {TM }}$ DTT (Dithiothreitol) (ThermoFisher Scientific, catalog number: 20290, storage temperature: $4^{\circ} \mathrm{C}$ )

12. Ni-NTA Agarose (25 ml) (QIAGEN, catalog number: 30210 , storage temperature: $4{ }^{\circ} \mathrm{C}$ )

13. Amicon ${ }^{\circledR}$ Ultra-4 Centrifugal Filter Unit (Merck-Millipore, catalog number: UFC801008, storage temperature: RT)

14. Imidazole for molecular biology (Applichem, catalog number: A1378,0050, storage temperature: RT)

15. Kinase assay solution (10x) (see Recipes) 


\section{Equipment}

1. Water bath (Julabo, ED-13 Open Heating Bath Circulator/discontinued product)

2. GloMax $^{\circledR} 20 / 20$ Luminometer (Promega, model/catalog number: 2030-100/E5311)

3. Eppendorf 5417R Refrigerated Centrifuge (Marshall Scientific, Product code: EP-5417R)

\section{Software}

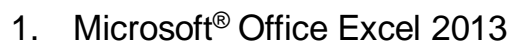

2. GraphPad Prism v6 software

\section{Procedure}

A. Preparation of Kinase-Glo ${ }^{\circledR}$ reagent

1. Mix the $10 \mathrm{ml}$ Kinase $\mathrm{Glo}{ }^{\circledR}$ Buffer with the lyophilized Kinase $\mathrm{Glo}{ }^{\circledR}$ Substrate.

2. Make sure that the dilution of the substrate powder is complete and the solution is clear.

3. Aliquot the Kinase-Glo ${ }^{\circledR}$ reagent and store it at $-20^{\circ} \mathrm{C}$ until usage.

B. Preparation of kinase assay solution $10 x$

Prepare the kinase assay solution $10 \mathrm{x}$ as described in the Recipes, aliquot and store it at $-20{ }^{\circ} \mathrm{C}$ until usage.

C. Determination of the TbGSK-3s fraction with the highest enzymatic activity

1. Perform the kinase assay for the different isolated fractions of the kinase (TbGSK-3s). The isolated fractions have been purified on $\mathrm{Ni}^{+2}$-nitrilotriacetate (Ni-NTA) agarose according to the manufacturer's instructions (Qiagen) with elutions of different imidazole concentrations (Fractions A, B, C and D using 50, 150, 250 and $400 \mathrm{nM}$ imidazole respectively) as previously described (Efstathiou et al., 2019).

Note: Isolated fractions of the TbGSK-3s were subsequently concentrated using Amicon ${ }^{\circledR}$ Ultra4 Centrifugal Filters and rediluted in kinase assay solution $1 x$ in order to remove excess imidazole from the samples. Imidazole in high concentrations could interfere during the kinase assay providing false results.

2. Adjust the temperature of the water bath to $30^{\circ} \mathrm{C}$.

3. Defreeze the Kinase-Glo ${ }^{\circledR}$ reagent from $-20^{\circ} \mathrm{C}$ to $4{ }^{\circ} \mathrm{C}$.

4. Defreeze the kinase assay solution $10 x$ from $-20^{\circ} \mathrm{C}$ to $4^{\circ} \mathrm{C}$.

5. Prepare master mix solution (containing a final concentration of $1 x$ kinase assay solution, 10 $\mu \mathrm{M}$ ATP, $10 \mu \mathrm{M}$ GSK-3 peptide substrate) as described below (final volume $40 \mu \mathrm{l}$ per sample): $4 \mu \mathrm{l}{ }^{*}$ [number of samples] of $10 x$ kinase assay solution $1 \mu \mathrm{l} *$ [number of samples] of $400 \mu \mathrm{M}$ ATP 
$1.2 \mu{ }^{*}$ [number of samples] of $333 \mu \mathrm{M}$ GSK-3 peptide substrate

$23.8 \mu{ }^{*}$ [number of samples] of $\mathrm{ddH}_{2} \mathrm{O}$

6. Mix well the master mix by pipetting up and down.

7. Add $30 \mu \mathrm{l}$ of the master mix in each Eppendorf.

8. Add $10 \mu \mathrm{l}$ of each TbGSK-3s isolated fraction (A, B, C and D fractions) in each tube.

9. Make duplicates or triplicates for each fraction.

10. Mix well the samples by pipetting up and down.

11. Spin down the samples.

12. Incubate the samples for $30 \mathrm{~min}$ in the water bath at $30^{\circ} \mathrm{C}$.

13. Stop the reaction by adding $40 \mu$ of cold Kinase-Glo ${ }^{\circledR}$ reagent $\left(4^{\circ} \mathrm{C}\right)$.

14. Spin down the samples.

15. Measure the luminescence in the GloMax ${ }^{\circledR} 20 / 20$ Luminometer for $1 \mathrm{~s}$.

Note: Read the luminescence with the 'ready to use' Promega protocol named 'Kinase-Glo'.

16. Determine the elution fraction of TbGSK-3s with the optimal enzymatic activity (see Data analysis $\mathrm{A}$ ).

17. Use the kinase immediately for the kinases assays or store it in a $50 \%(\mathrm{v} / \mathrm{v})$ glycerol stock at $80^{\circ} \mathrm{C}$.

Notes:

a. Purified elution fraction C of TbGSK-3s (initially eluted with $250 \mathrm{mM}$ imidazole) was determined in our analysis as the one with higher enzymatic activity (see Data analysis $A$ ).

b. It is recommended to use the kinase TbGSK-3s fractions immediately after the elution to ensure maximum activity. The $50 \%(\mathrm{~V} / \mathrm{v})$ glycerol stock of the kinase has diminished activity depending on the time period that it is stored at $-80{ }^{\circ} \mathrm{C}$ and they should be used up to 3-5 days upon isolation to avoid complete loss of the kinase activity.

D. Determination of the optimal protein kinase (TbGSK-3s) concentration

1. Perform the kinase assay with the appropriate elution fraction (elution fraction C) of TbGSK-3s which was identified above as the one with higher enzymatic activity.

2. Follow Steps C2-C4.

3. Prepare master mix solution (containing a final concentration of $1 \mathrm{x}$ kinase assay solution, 10 $\mu \mathrm{M}$ ATP, $10 \mu \mathrm{M}$ GSK-3 peptide substrate) as described below (final volume $40 \mu \mathrm{l}$ per sample):

$4 \mu \mathrm{l} *$ [number of samples] of 10x kinase assay solution

$1 \mu \mathrm{l}{ }^{*}$ [number of samples] of $400 \mu \mathrm{M}$ ATP

$1.2 \mu \mathrm{l}{ }^{*}$ [number of samples] of $333 \mu \mathrm{M}$ GSK-3 peptide substrate

$23.8 \mu{ }^{*}$ [number of samples] of $\mathrm{ddH}_{2} \mathrm{O}$

4. Follow Steps C6-C7.

5. Add $10 \mu$ l of TbGSK-3s isolated fraction $C$ in different concentrations (containing $0,2,5,10,15$, 20, 40, 100 and $200 \mathrm{ng}$ TbGSK-3s, diluted with $\mathrm{dd}_{2} \mathrm{O}$ ).

Note: Different concentrations of kinase can be used if needed, depending on the parameters 
of the experiment.

6. Follow Steps C9-C15.

7. Determine the optimal concentration of TbGSK-3s for the kinase assays (see Data analysis B). Note: $40 \mathrm{ng}$ of TbGSK-3s (Fraction C) was determined in our analysis as the optimal concentration of TbGSK-3s for the kinase assays (see Data analysis B). Fraction C contains $20 \mathrm{ng} / \mu \mathrm{l}$ of TbGSK3s (see Data analysis B).

E. Determination of the ATP concentration

1. Perform the kinase assay with the TbGSK-3s isolated fraction C.

2. Follow Steps C2-C4.

3. Prepare master mix solution (containing a final concentration of $1 \times$ kinase assay solution, $40 \mathrm{ng}$ of TbGSK-3s isolated fraction C, $10 \mu \mathrm{M}$ GSK-3 peptide substrate) as described below (final volume $40 \mu$ l per sample):

$4 \mu \mathrm{l}{ }^{*}$ [number of samples] of 10x kinase assay solution

$2 \mu \mathrm{l}$ * [number of samples] of TbGSK-3s isolated fraction C

$1.2 \mu{ }^{*}$ [number of samples] of $333 \mu \mathrm{M}$ GSK-3 peptide substrate

$23.8 \mu \mathrm{l}$ * [number of samples] of $\mathrm{ddH}_{2} \mathrm{O}$

4. Follow Steps C6-C7.

5. Add $10 \mu \mathrm{l}$ of ATP in different concentrations of $(0,0.5,1,2,5,7.5,10$ and $50 \mu \mathrm{M})$. Note: Different concentrations of ATP can be used if needed, depending on the parameters of the experiment. However, for concentrations of ATP up to $10 \mu \mathrm{M}$, you can use the Kinase-Glo ${ }^{\circledR}$ Assay (Promega), for 0-100 $\mu \mathrm{M}$ of ATP, you can use the Kinase-Glo ${ }^{\circledR}$ Plus Assay (Promega) and for 0-500 $\mu$ M, you can use the Kinase-Glo ${ }^{\circledR}$ Max Assay (Promega).

6. Follow Steps C9-C15.

7. Plot the diagram of the rate of reaction DY/DX versus the ATP concentration and determine the Vmax and Km of ATP (see Data analysis C).

Notes:

a. $D Y=\left[\left(R L U \times 10^{6}\right.\right.$ upon 30 min of reaction $)-\left(R L U \times 10^{6}\right.$ upon 0 min of reaction $\left.)\right]$

b. $D X=$ time of reaction $=30 \mathrm{~min}$

c. Vmax is the maximum rate of reaction and represents the rate of reaction when the enzyme is saturated with substrate.

d. The value of the Michaelis-Menten constant $(\mathrm{Km})$ is numerically equal to the substrate concentration at which the reaction rate is half of Vmax.

e. $\mathrm{Km}$ of ATP was determined equal to $6.3 \mu \mathrm{M}$ using $40 \mathrm{ng}$ of TbGSK-3s fraction C (see Data analysis C).

f. A standard ATP concentration, identical in all kinase assays performed with TbGSK-3s, was used and it was equal to the Km for the ATP $(6.3 \mu \mathrm{M})$. 
F. Determination of the GSK-3 peptide substrate concentration

1. Perform the kinase assay with the TbGSK-3s isolated fraction C.

2. Follow Steps C2-C4.

3. Prepare master mix solution (containing a final concentration of $1 \mathrm{x}$ kinase assay solution, $40 \mathrm{ng}$ of TbGSK-3s isolated fraction C, $10 \mu \mathrm{M}$ ATP) as described below (final volume $40 \mu$ per sample): $4 \mu \mathrm{l}$ [number of samples] of 10x kinase assay

$2 \mu{ }^{*}$ [number of samples] of TbGSK-3s isolated fraction C

$1.2 \mu \mathrm{l}{ }^{*}$ [number of samples] of $400 \mu \mathrm{M}$ ATP

$23.8 \mu \mathrm{l}$ * [number of samples] of $\mathrm{ddH}_{2} \mathrm{O}$

4. Follow Steps C6-C7.

5. Add $10 \mu$ l of GSK-3 substrate in different concentrations of $(0,1,2,5,10$ and $50 \mu \mathrm{M})$. Note: Different concentrations of substrate can be used if needed, depending on the parameters of the experiment.

6. Follow Steps C9-C15.

7. Plot the diagram of the rate of reaction DY/DX versus the GSK-3 concentration and determine the Vmax and Km of GSK-3 substrate (see Data analysis C).

Notes:

a. Km of GSK-3 substrate was determined equal to $5.8 \mu \mathrm{M}$ using $40 \mathrm{ng}$ of TbGSK-3s (Fraction C) (see Data analysis $C$ ).

b. A standard GSK-3 substrate concentration, identical in all kinase assays performed with TbGSK3 s, was used and it was equal to the Km for the GSK-3 substrate (5.8 $\mu \mathrm{M})$.

G. Identification of TbGSK-3s inhibitors

1. Perform the kinase assay with the TbGSK-3s isolated fraction $C$.

2. Follow Steps C2-C4.

3. Prepare master mix solution (containing a final concentration of $1 \mathrm{x}$ kinase assay solution, $40 \mathrm{ng}$ of TbGSK-3s isolated fraction C) as described below (final volume $40 \mu$ per sample):

$4 \mu{ }^{*}$ [number of samples] of 10x kinase assay solution

$2 \mu \mathrm{l}$ * [number of samples] of TbGSK-3s isolated fraction C

$22.7 \mu \mathrm{l} *$ [number of samples] of $\mathrm{ddH}_{2} \mathrm{O}$

4. Follow Steps C6-C7.

5. Add $10 \mu \mathrm{l}$ of inhibitor in different concentrations $(0,0.01,0.03,0.1,0.33,1$ and $3.33 \mu \mathrm{M})$. Note: Different concentrations of inhibitors can be used if needed, depending on the parameters of the experiment.

6. Add $5.8 \mu \mathrm{M}$ GSK-3 peptide substrate in each sample ( $0.7 \mu \mathrm{l}$ of $333 \mu \mathrm{M}$ GSK-3 peptide substrate).

7. Add $6.3 \mu \mathrm{M}$ ATP in each sample $(0.6 \mu \mathrm{l}$ of $400 \mu \mathrm{M}$ ATP $)$.

8. Follow Steps C9-C15.

9. Plot the diagram of the \% inhibition of TbGSK-3s activity versus the inhibitor's concentration and determine the $\mathrm{IC}_{50}$ of the inhibitor (see Data analysis $\mathrm{D}$ ). 
Note: The sample with no inhibitor serves as a positive control. Moreover, use a sample that contains no ATP as a negative control ( $4 \mu$ l kinase assay solution 10x, $2 \mu$ l TbGSK-3s isolated fraction $C$ containing $40 \mathrm{ng}$ of kinase, $0.7 \mu \mathrm{l}$ GSK-3 peptide substrate $333 \mu \mathrm{M}$ in order to obtain $5.8 \mu \mathrm{M}$ final concentration, and $\left.33.3 \mu \mathrm{ldd} \mathrm{H}_{2} \mathrm{O}\right)$.

\section{Data analysis}

A. Determination of the TbGSK-3s fraction with enzymatic activity:

1. The fraction that contains the active kinase will utilize the ATP to phosphorylate the GSK-3 substrate and therefore the concentration of the ATP in the sample will reduce. As a result, the luciferin will no longer have the same amount of ATP to interact with and the reaction will produce less light. Concequently, the luminescence (RLU) measurement of the sample will be lower than the one of the negative control (sample containing no kinase).

2. Collect the luminescence readings for the elution fractions of TbGSK-3s.

3. Calculate the average value of luminescence and the standard deviation for each fraction.

4. Plot the diagram of the luminescence values (Figure 2).

5. The fraction with the lowest value of luminescence is the one with the optimal enzymatic activity and can be used for further experiments for kinase assays.

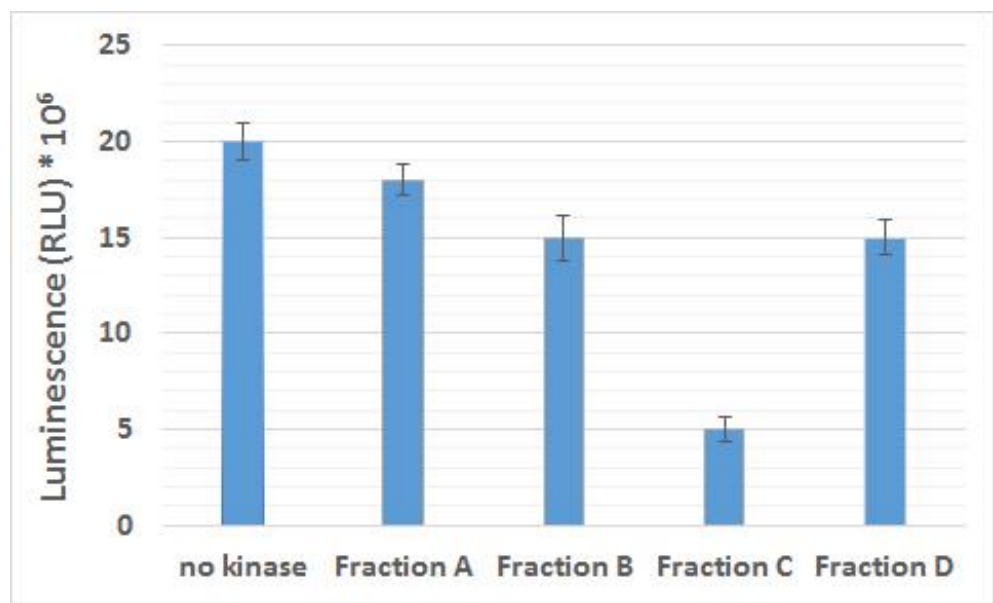

Figure 2. Luminescence values for isolated TbGSK-3s fractions $A, B, C$ and $D$. The TbGSK-3s fractions A, B, C and D were purified on $\mathrm{Ni}^{+2}$-nitrilotriacetate (Ni-NTA) resin according to the manufacturer's instructions (Macherey-Nagel) with elutions of different imidazole concentrations (Fractions A, B, C and D using 50, 150, 250 and $400 \mathrm{nM}$ imidazole respectively) as previously described (Efstathiou et al., 2019) and subsequently they were concentrated using Amicon ${ }^{\circledR}$ Ultra $0.5 \mathrm{ml}$ Centrifugal Filters and re-diluted in kinase assay solution $1 \mathrm{x}$ in order to remove excess imidazole from the samples. Kinase assay solution $1 \mathrm{x}$ (no kinase) was used as a negative control. 
B. Determination of the optimal protein kinase (TbGSK-3s) concentration

1. Determine the amount of kinase present in each fraction using Bradford protein assay (He, 2011) and by a semi-quantification method based on the intensity of the signal in the Western blot upon comparison with already known amount of protein in the marker bands and given that the volume of the kinase fraction put in the Western blot is constant and known.

Notes:

a. The protocol and analysis procedure for the Western blot, including the reagents and the materials used, can be found in bibliography (Mahmood and Yang, 2012; Heiber et al., 2014).

b. The Bradford protein assay is used for the quantification of the total protein amount in each eluted fraction, while the Western plot was used in order to illustrate the existence of TbGSK-3s in each fraction.

2. Isolated fraction $C$ (Lane 5 in Figure 3-TbGSK-3s fraction which was eluted from the $\mathrm{Ni}^{+2}$ nitrilotriacetate (Ni-NTA) resin $\mathrm{Ni}^{2+}$ beads using $250 \mathrm{mM}$ imidazole) was estimated to contain 20 $\mathrm{ng} / \mathrm{\mu l}$ of TbGSK-3s (Figure 3).

3. Collect the luminescence readings for the samples containing different concentrations of TbGSK-3s.

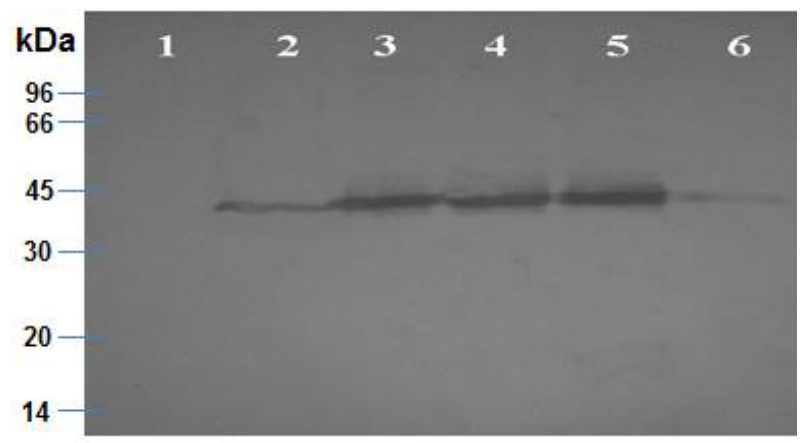

Figure 3. Western blot of isolated TbGSK-3s fractions A, B, C and D. $30 \mu$ of TbGSK-3s fractions A. (lane 3), B. (lane 4), C. (lane 5) and D. (lane 6), which were purified on $\mathrm{Ni}^{+2}$ nitrilotriacetate (Ni-NTA) resin with elutions of different imidazole concentrations (50, 150, 250 and $400 \mathrm{nM}$ imidazole respectively), and the remaining $\mathrm{Ni}^{+2}$-nitrilotriacetate (Ni-NTA) resin upon the elutions (lane 2), were identified in a Western blot using an affinity purified polyclonal antibody raised against LGSK-3s. The Western blot showed a unique band at the theoretical molecular mass of TbGSK-3s (40 kDa). In lane 1, $30 \mu$ l of kinase assay solution $1 \mathrm{x}$ were also used as a negative control. 
Table 1. TbGSK-3s used in kinase assay, expressed in $\mathrm{ng}$, when using different volumes of isolated fraction $C$. In the column entitled 'Fraction $C$ ', the volume of the eluted kinase TbGSK-3s (expressed in $\mu$ ) which was added in each sample, is given. In the column entitled 'TbGSK-3s', the corresponding concentration of the kinase that corresponds to the volume of Fraction $\mathrm{C}$ added in each sample, is calculated. The calculation of the corresponding kinase concentration was based on the protein concentration provided by Bradford protein assay as mentioned in paragraph B1 of Data Analysis Section.

\begin{tabular}{ll}
\hline Fraction C $(\boldsymbol{\mu l})$ & TbGSK-3s $(\mathbf{n g})$ \\
\hline 0 & 0 \\
0.1 & 2 \\
0.25 & 5 \\
0.5 & 10 \\
0.75 & 15 \\
1 & 20 \\
2 & 40 \\
5 & 100 \\
10 & 200 \\
\hline
\end{tabular}

4. Calculate the average value of luminescence for each TbGSK-3s concentration (the concentration of TbGSK-3s in each sample depending on the added volume of fraction $C(\mu l)$ is shown in Table 1).

5. Plot the diagram of the luminescence values versus the amount of TbGSK-3s containing in the sample (Figure 4).

6. The sample which diminishes the luminescence value at the highest portion while it has not yet been saturated is circled in red in the plot and it is the one containing $2 \mu$ of fraction C (40 ng TbGSK-3s) (Figure 4).

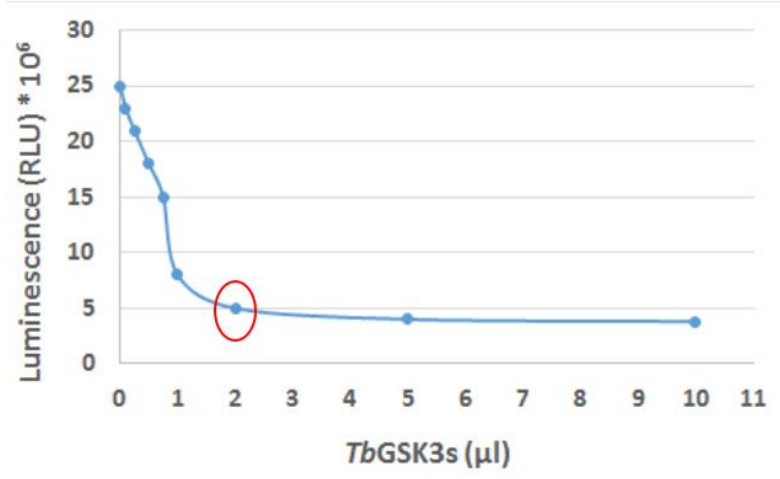

Figure 4. Plot of luminescence values versus the amount of TbGSK-3s. Luminescence (RLU) in the $Y$ axis represents the value of each sample when measured in the GloMax ${ }^{\circledR}$ 20/20 Luminometer and it is expressed in $\mathrm{RLU}^{*} 10^{6}$. The $\mathrm{X}$ axis represents the volume of the kinase ( $T$ bGSK-3s) in $\mu \mathrm{l}$ wich is added in each sample. 
C. Determination of the Km for ATP and GSK-3 substrate

1. Collect the luminescence readings for the samples containing different concentrations of GSK3 substrate or ATP.

2. Calculate the average value of luminescence $(R L U)$ and the standard deviation for each sample.

3. Calculate the rate of reaction DY/DX for each sample (as shown in Table 2 for the ATP and in Table 3 for the GSK-3 substrate),

where $D Y=\left[\left(R L U \times 10^{6}\right.\right.$ upon $30 \mathrm{~min}$ of reaction $)-\left(R L U \times 10^{6}\right.$ upon 0 min of reaction $\left.)\right]$

$\mathrm{DX}=$ time of reaction $=30 \mathrm{~min}$

Table 2. Calculations for the rate of reaction DY/DX for different concentrations of ATP

\begin{tabular}{|c|c|c|c|c|c|}
\hline ATP $(\mu \mathrm{M})$ & $\begin{array}{l}\text { RLUa } \\
{\left[R L U \times 10^{6}\right.} \\
\text { t0 = } 0 \mathrm{~min}]\end{array}$ & $\begin{array}{l}R^{b} U^{b} \\
{\left[R L U \times 10^{6}\right.} \\
t 1=30 \mathrm{~min}]\end{array}$ & $\begin{array}{l}\text { DY } \\
\left(R L U^{a}-\right. \\
\left.R L U^{b}\right)\end{array}$ & $\begin{array}{l}\text { DX } \\
\text { (min of } \\
\text { reaction) }\end{array}$ & $\begin{array}{l}\text { Rate of } \\
\text { reaction } \\
\text { (DY/DX) }\end{array}$ \\
\hline 0 & 0 & 0 & 0 & 30 & 0 \\
\hline 0.5 & 2.52 & 1.21 & 1.31 & 30 & 0.044 \\
\hline 1 & 6.31 & 0.86 & 5.45 & 30 & 0.182 \\
\hline 2 & 9.38 & 3.38 & 6.00 & 30 & 0.200 \\
\hline 5 & 18.81 & 11.61 & 7.20 & 30 & 0.240 \\
\hline 7.5 & 23.66 & 15.26 & 8.40 & 30 & 0.280 \\
\hline 10 & 28.84 & 19.84 & 9.00 & 30 & 0.300 \\
\hline 20 & 68.37 & 54.33 & 14.04 & 30 & 0.468 \\
\hline 50 & 121.65 & 106.30 & 15.350 & 30 & 0.512 \\
\hline
\end{tabular}

RLU stands for luminescence. ${ }^{a}$ is for the RLU (luminescence) measured in the beginning of the reaction (t0 $=0 \mathrm{~min}$ ). ${ }^{\mathrm{b}}$ is for the $\mathrm{RLU}$ (luminescence) measured in the end of the reaction ( $\mathrm{t} 1=$ $30 \mathrm{~min}$ ). DY is the difference between the luminescence measurements in the beginning and at the end of the reaction. DX represents the time of reaction between the two luminescence measurements (total time of reaction, $30 \mathrm{~min}$ ). 
Table 3. Calculations for the rate of reaction DY/DX for different concentrations of GSK3 substrate

\begin{tabular}{|c|c|c|c|c|c|}
\hline $\begin{array}{l}\text { GSK-3 } \\
\text { substrate } \\
(\mu \mathrm{M})\end{array}$ & $\begin{array}{l}R_{L} \mathbf{a}^{\mathrm{a}} \\
{\left[\mathrm{RLU} \times 10^{4}\right.} \\
\text { t0 =0 min] }\end{array}$ & $\begin{array}{l}\text { RLU }^{\mathrm{b}} \\
{\left[R L U \times 10^{4}\right.} \\
\mathrm{t} 1=30 \mathrm{~min}]\end{array}$ & $\begin{array}{l}\text { DY } \\
\left(R L U^{a}-\right. \\
\left.R L U^{b}\right)\end{array}$ & $\begin{array}{l}\text { DX } \\
(\min \text { of } \\
\text { reaction) }\end{array}$ & $\begin{array}{l}\text { Rate of } \\
\text { reaction } \\
\text { (DY/DX) }\end{array}$ \\
\hline 0 & 0 & 0 & 0 & 30 & 0 \\
\hline 1 & 29.36 & 24.86 & 4.50 & 30 & 0.150 \\
\hline 2 & 28.81 & 23.35 & 5.46 & 30 & 0.182 \\
\hline 5 & 26.44 & 19.84 & 6.60 & 30 & 0.220 \\
\hline 10 & 27.67 & 18.07 & 9.60 & 30 & 0.320 \\
\hline 20 & 28.69 & 14.59 & 14.10 & 30 & 0.470 \\
\hline 50 & 28.99 & 14.29 & 14.70 & 30 & 0.490 \\
\hline
\end{tabular}

RLU stands for luminescence. ${ }^{a}$ is for the RLU (luminescence) measured in the beginning of the reaction ( $\mathrm{t} 0=0 \mathrm{~min}$ ). ${ }^{\mathrm{b}}$ is for the RLU (luminescence) measured in the end of the reaction ( $\mathrm{t} 1=$ $30 \mathrm{~min})$. DY is the difference between the luminescence measurements in the beginning and at the end of the reaction. DX represents the time of reaction between the two luminescence measurements (total time of reaction, $30 \mathrm{~min}$ ).

4. Plot the diagram of the rate of reaction DY/DX versus the ATP concentration (Figure 5) and the GSK-3 substrate (Figure 6) concentration.

5. Determine the Vmax in the plot.

6. Find the $\mathrm{Vmax} / 2$ in the plot and determine the Km of ATP and of GSK-3 substrate.

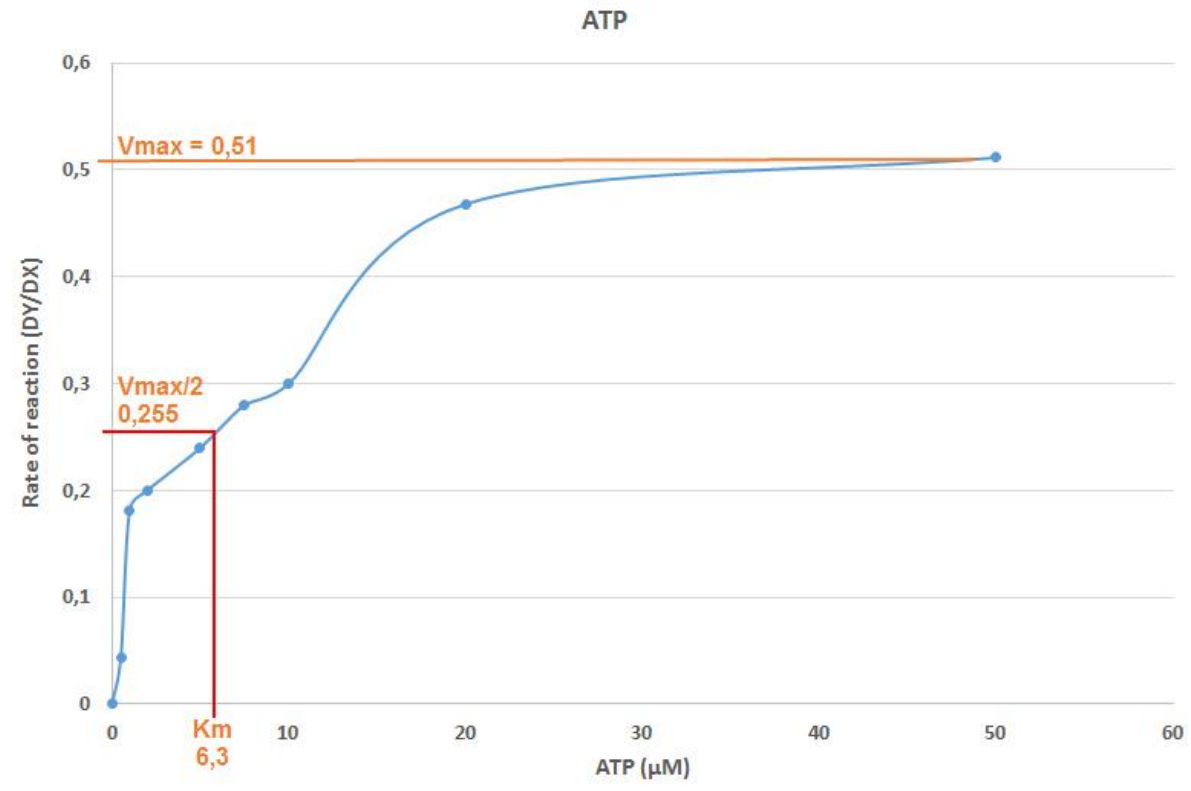

Figure 5. Plot of the reaction rate DY/DX versus the ATP concentration. $\ln X$ axis, the ATP concentration $(\mu \mathrm{M})$ for each sample is presented. In $Y$ axis, the reaction rate DY/DX that 
corresponds to each sample with different ATP concentration is presented, as calculated in Table 2. The Vmax for the reaction is calculated in the plot and it equals to $0.51(\mathrm{Vmax} / 2=$ 0.255). The Km for ATP after plotting the corresponding ATP concentration of the Vmax/2 value, was determined to be equal to $6.3 \mu \mathrm{M}$.

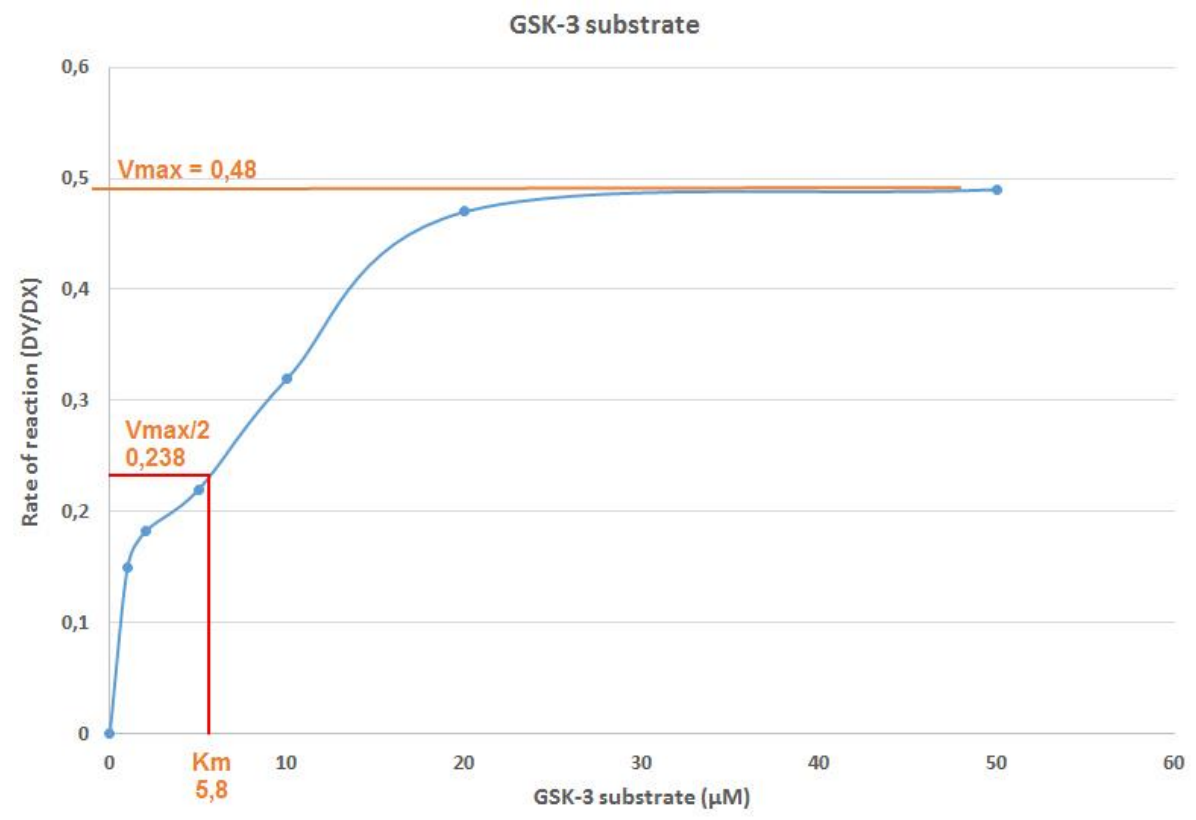

Figure 6. Plot of the reaction rate DY/DX versus the GSK-3 substrate concentration. In $\mathrm{X}$ axis, the GSK-3 substrate concentration $(\mu \mathrm{M})$ for each sample is presented. In $Y$ axis, the reaction rate DY/DX that corresponds to each sample with different GSK-3 substrate concentration is presented, as calculated in Table 3. The Vmax for the reaction is calculated in the plot and it equals to $0.48(\mathrm{Vmax} / 2=0.238)$. The Km for GSK-3 substrate after plotting the corresponding GSK-3 substrate concentration of the Vmax/2 value, was determined to be equal to $5.8 \mu \mathrm{M}$.

D. Identification of TbGSK-3s inhibitors and calculation of their IC $\mathrm{C}_{50}$ values

1. Collect the luminescence readings for the samples containing different concentations of each possible TbGSK-3s inhibitors.

2. Collect the luminescence readings for negative and positive control.

3. Calculate the average value of luminescence $(R L U)$ and the standard deviation for each sample.

4. Substract the average RLU value of the negative control from the average RLU value of all the samples and from the positive control.

5. The positive control's RLU value (after the substraction of the negative control RLU value) corresponds to $100 \%$ TbGSK-3s activity.

6. Calculate the \% inhibition of TbGSK-3s activity for each concentration of the inhibitor using the formula below:

$\%$ inhibition $=(R L U$ value of inhibitor/RLU value of positive control $) \times 100$ 
Note: The RLU values used in the formula are the ones after the substraction of the negative control RLU value.

7. Plot the diagram of the $\%$ inhibition of TbGSK-3s activity versus the inhibitor's concentration. Indicative plots for an analog with no inhibition and for analogs presenting weak, moderate and strong activity against the TbGSK-3s are shown in Figures 7, 8, 9 and 10 respectively.

8. Determine the $I C_{50}$ value of the inhibitor. The $I C_{50}$ value represents the concentration of the inhibitor that inhibits the kinase activity at a 50\% rate, therefore the concentration of the inhibitor that increases $50 \%$ of the sample's luminescence compared to the positive sample (positive control is the sample that contains no inhibitor, but contains the kinase, the GSK-3 substrate and the ATP).

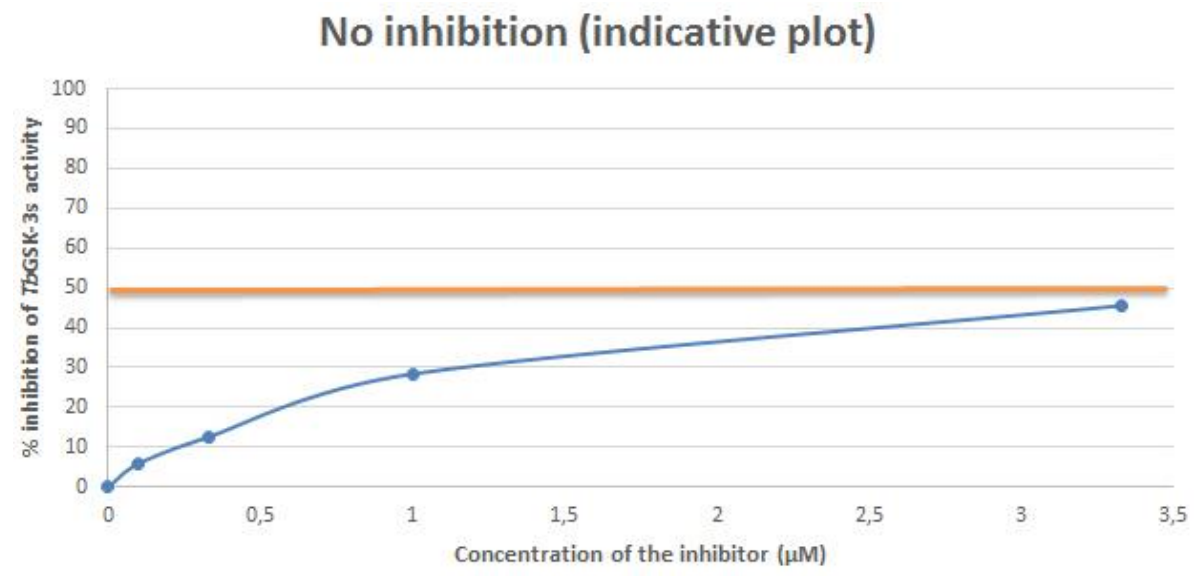

Figure 7. Indicative plot of an analog with no inhibitory activity against TbGSK-3s. In X axis, the concentration of the inhibitor $(\mu \mathrm{M})$ in each sample, is displayed. In $Y$ axis, the \% inhibition of TbGSK-3s activity is expressed. The $\mathrm{IC}_{50}$ (in $\mu \mathrm{M}$ ) is calculated in the plot by corresponding the concentration of the inhibitor that causes 50\% inhibition of the TbGSK-3s activity. The $\mathrm{IC}_{50}$ of the compound used for the current indicative plot is calculated $>3.5 \mu \mathrm{M}$.

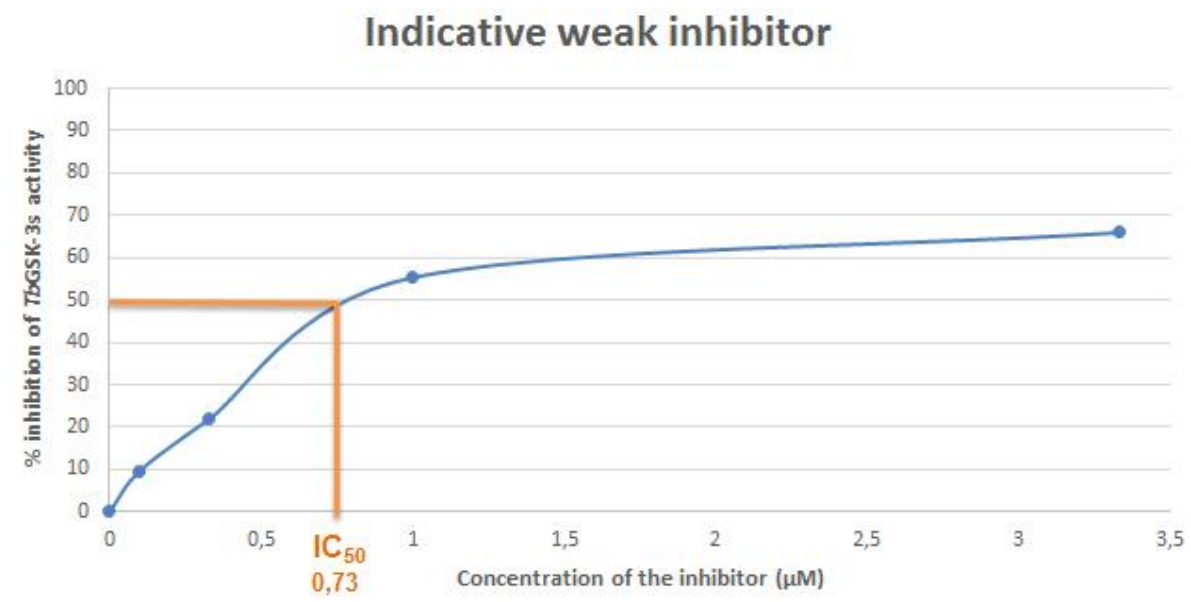

Figure 8. Indicative plot of an analogue with weak inhibitory activity against TbGSK-3s. In $X$ axis, the concentration of the inhibitor $(\mu \mathrm{M})$ in each sample, is displayed. In $Y$ axis, the \% 
inhibition of TbGSK-3s activity is expressed. The $\mathrm{IC}_{50}$ (in $\mu \mathrm{M}$ ) is calculated in the plot by corresponding the concentration of the inhibitor that causes 50\% inhibition of the TbGSK-3s activity. The $\mathrm{IC}_{50}$ of the compound used for the current indicative plot is calculated $0.73 \mu \mathrm{M}$.

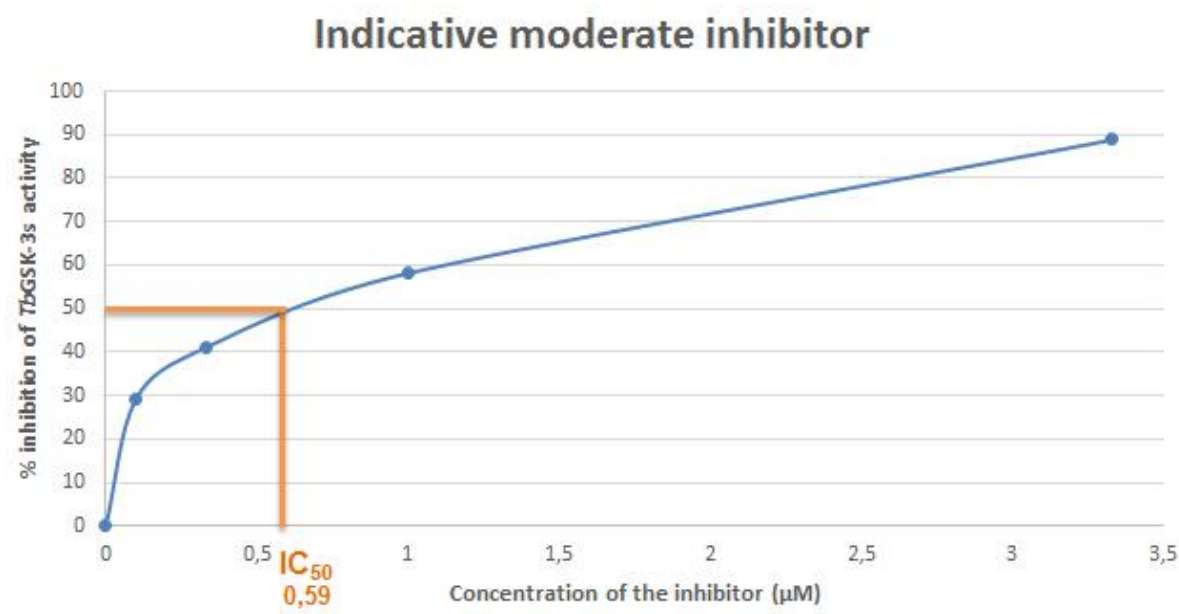

Figure 9. Indicative plot of an analogue with modearte inhibitory activity against TbGSK-

3s. In $\mathrm{X}$ axis, the concentration of the inhibitor $(\mu \mathrm{M})$ in each sample, is displayed. In $\mathrm{Y}$ axis, the \% inhibition of TbGSK-3s activity is expressed. The $\mathrm{IC}_{50}$ (in $\mu \mathrm{M}$ ) is calculated in the plot by corresponding the concentration of the inhibitor that causes 50\% inhibition of the TbGSK-3s activity. The $\mathrm{IC}_{50}$ of the compound used for the current indicative plot is calculated $0.59 \mu \mathrm{M}$.

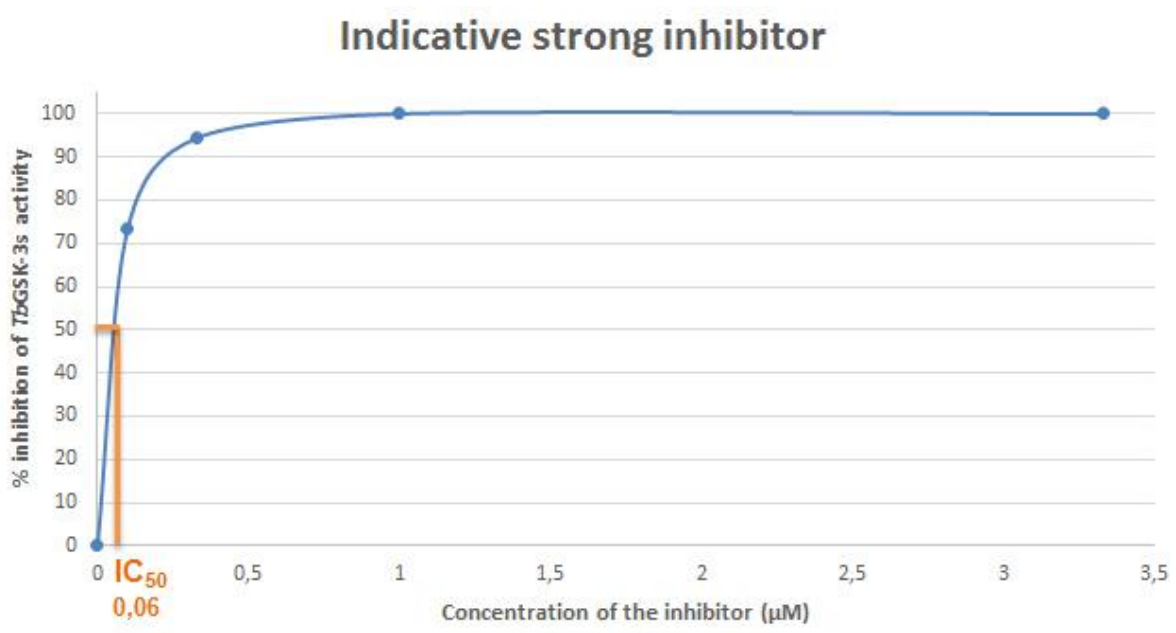

Figure 10. Indicative plot of an analog with strong inhibitory activity against TbGSK-3s. In $X$ axis, the concentration of the inhibitor $(\mu \mathrm{M})$ in each sample, is displayed. In $Y$ axis, the \% inhibition of TbGSK-3s activity is expressed. The $\mathrm{IC}_{50}$ (in $\mu \mathrm{M}$ ) is calculated in the plot by corresponding the concentration of the inhibitor that causes 50\% inhibition of the TbGSK-3s activity. The $\mathrm{IC}_{50}$ of the compound used for the current indicative plot is calculated $0.06 \mu \mathrm{M}$. 


\section{$\underline{\text { Recipes }}$}

1. Kinase assay solution (10x)

MOPS (pH = 7.5) $500 \mathrm{mM}$

$\mathrm{MgCl}_{2} 200 \mathrm{mM}$

EGTA $100 \mathrm{mM}$

DTT $20 \mathrm{mM}$

Note: Aliquot in Eppendorfs and store at $-20^{\circ} \mathrm{C}$.

\section{Acknowledgements}

The authors would like to thank Prof. George A.M. Cross (Rockefeller University, New York) for donating the T. brucei BSF 90-13 strain, Prof. Pevelope Mavromara for her advice and support on the baculovirus expression system. We would also like to thank for financial support IKY-SIEMENS Postdoctoral scholarship of excellence 2016-2017 'contract number 2016-017-0173-10398' and the COST Action BM0801. This protocol is based on the TbGSK-3s kinase assay presented in (Efstathiou et al., 2019) and is a modified version of the kinase assay protocol performed against leismanial GSK-3s in Xingi et al., 2009.

\section{Competing interests}

Both authors declare that they have no conflicts of interest.

\section{References}

1. Cohen, P. (1999). The development and therapeutic potential of protein kinase inhibitors. Curr Opin Chem Biol 3(4): 459-465.

2. Cohen, P. and Goedert, M. (2004). GSK3 inhibitors: development and therapeutic potential. Nat Rev Drug Discov 3(6): 479-487.

3. Efstathiou, A., Gaboriaud-Kolar, N., Myrianthopoulos, V., Vougogiannopoulou, K., Subota, I., Aicher, S., Mikros, E., Bastin, P., Skaltsounis, A. L., Soteriadou, K. and Smirlis, D. (2019). Indirubin analogues inhibit trypanosoma brucei glycogen synthase kinase 3 short and t. brucei growth. Antimicrob Agents Chemother 63(6).

4. Ferandin, Y., Bettayeb, K., Kritsanida, M., Lozach, O., Polychronopoulos, P., Magiatis, P., Skaltsounis, A. L. and Meijer, L. (2006). 3'-Substituted 7-halogenoindirubins, a new class of cell death inducing agents. J Med Chem 49(15): 4638-4649.

5. Gaboriaud-Kolar, N., Vougogiannopoulou, K. and Skaltsounis, A. L. (2015). Indirubin derivatives: a patent review (2010 - present). Expert Opin Ther Pat 25(5): 583-593.

6. He, F. (2011). Bradford protein assay. Bio-101: e45. 
Please cite this article as: Efstathiou and Smirlis, (2020). A Radioactive-free Kinase Inhibitor Discovery Assay Against the Trypanosoma brucei Glycogen Synthase Kinase-3 short (TbGSK-3s),Bio-protocol 10 (2): e3493. DOI: 10.21769/BioProtoc.3493.

7. Heiber, A. and Spielmann, T. (2014). Preparation of parasite protein extracts and Western blot analysis. Bio-protocol 4(11): e1136.

8. Hoessel, R., Leclerc, S., Endicott, J. A., Nobel, M. E., Lawrie, A., Tunnah, P., Leost, M., Damiens, E., Marie, D., Marko, D., Niederberger, E., Tang, W., Eisenbrand, G. and Meijer, L. (1999). Indirubin, the active constituent of a Chinese antileukaemia medicine, inhibits cyclin-dependent kinases. Nat Cell Biol 1(1): 60-67.

9. Lilienthal, E., Kolanowski, K. and Becker, W. (2010). Development of a sensitive non-radioactive protein kinase assay and its application for detecting DYRK activity in Xenopus laevis oocytes. BMC Biochem 11: 20.

10. Mahmood, T. and Yang, P. C. (2012). Western blot: technique, theory, and trouble shooting. $N$ Am J Med Sci 4(9): 429-434.

11. Manning, G., Whyte, D. B., Martinez, R., Hunter, T. and Sudarsanam, S. (2002). The protein kinase complement of the human genome. Science 298(5600): 1912-1934.

12. Masch, A. and Kunick, C. (2015). Selective inhibitors of Plasmodium falciparum glycogen synthase-3 (PfGSK-3): New antimalarial agents? Biochim Biophys Acta 1854(10 Pt B): 16441649.

13. Mazitschek, R. and Giannis, A. (2004). Inhibitors of angiogenesis and cancer-related receptor tyrosine kinases. Curr Opin Chem Biol 8(4): 432-441.

14. Meijer, L., Skaltsounis, A. L., Magiatis, P., Polychronopoulos, P., Knockaert, M., Leost, M., Ryan, X. P., Vonica, C. A., Brivanlou, A., Dajani, R., Crovace, C., Tarricone, C., Musacchio, A., Roe, S. M., Pearl, L. and Greengard, P. (2003). GSK-3-selective inhibitors derived from Tyrian purple indirubins. Chem Biol 10(12): 1255-1266.

15. Myrianthopoulos, V., Kritsanida, M., Gaboriaud-Kolar, N., Magiatis, P., Ferandin, Y., Durieu, E., Lozach, O., Cappel, D., Soundararajan, M., Filippakopoulos, P., Sherman, W., Knapp, S., Meijer, L., Mikros, E. and Skaltsounis, A. L. (2013). Novel inverse binding mode of indirubin derivatives yields improved selectivity for dyrk kinases. ACS Med Chem Lett 4(1): 22-26.

16. Oduor, R. O., Ojo, K. K., Williams, G. P., Bertelli, F., Mills, J., Maes, L., Pryde, D. C., Parkinson, T., Van Voorhis, W. C. and Holler, T. P. (2011). Trypanosoma brucei glycogen synthase kinase3, a target for anti-trypanosomal drug development: a public-private partnership to identify novel leads. PLoS Negl Trop Dis 5(4): e1017.

17. Ojo, K. K., Gillespie, J. R., Riechers, A. J., Napuli, A. J., Verlinde, C. L., Buckner, F. S., Gelb, M. H., Domostoj, M. M., Wells, S. J., Scheer, A., Wells, T. N. and Van Voorhis, W. C. (2008). Glycogen synthase kinase 3 is a potential drug target for African trypanosomiasis therapy. Antimicrob Agents Chemother 52(10): 3710-3717.

18. Polychronopoulos, P., Magiatis, P., Skaltsounis, A. L., Myrianthopoulos, V., Mikros, E., Tarricone, A., Musacchio, A., Roe, S. M., Pearl, L., Leost, M., Greengard, P. and Meijer, L. (2004). Structural basis for the synthesis of indirubins as potent and selective inhibitors of glycogen synthase kinase-3 and cyclin-dependent kinases. J Med Chem 47(4): 935-946. 
Please cite this article as: Efstathiou and Smirlis, (2020). A Radioactive-free Kinase Inhibitor Discovery Assay Against the Trypanosoma brucei Glycogen Synthase Kinase-3 short (TbGSK-3s),Bio-protocol 10 (2): e3493. DOI: 10.21769/BioProtoc.3493.

19. Resnick, L. and Fennell, M. (2004). Targeting JNK3 for the treatment of neurodegenerative disorders. Drug Discov Today 9(21): 932-939.

20. Swinney, Z. T., Haubrich, B. A., Xia, S., Ramesha, C., Gomez, S. R., Guyett, P., Mensa-Wilmot, K. and Swinney, D. C. (2016). A four-point screening method for assessing molecular mechanism of action (MMOA) identifies tideglusib as a time-dependent inhibitor of trypanosoma brucei GSK3ß. PLoS Negl Trop Dis 10(3): e0004506.

21. Urich, R., Grimaldi, R., Luksch, T., Frearson, J. A., Brenk, R. and Wyatt, P. G. (2014). The design and synthesis of potent and selective inhibitors of Trypanosoma brucei glycogen synthase kinase 3 for the treatment of human african trypanosomiasis. J Med Chem 57(18): 7536-7549.

22. Vougogiannopoulou, K., Ferandin, Y., Bettayeb, K., Myrianthopoulos, V., Lozach, O., Fan, Y., Johnson, C. H., Magiatis, P., Skaltsounis, A. L., Mikros, E. and Meijer, L. (2008). Soluble 3',6substituted indirubins with enhanced selectivity toward glycogen synthase kinase -3 alter circadian period. J Med Chem 51(20): 6421-6431.

23. Ways, D. K. and Sheetz, M. J. (2000). The role of protein kinase $C$ in the development of the complications of diabetes. Vitam Horm 60: 149-193.

24. Woodland, A., Grimaldi, R., Luksch, T., Cleghorn, L. A., Ojo, K. K., Van Voorhis, W. C., Brenk, R., Frearson, J. A., Gilbert, I. H. and Wyatt, P. G. (2013). From on-target to off-target activity: identification and optimisation of Trypanosoma brucei GSK3 inhibitors and their characterisation as anti-Trypanosoma brucei drug discovery lead molecules. ChemMedChem 8(7): 1127-1137.

25. Xingi, E., Smirlis, D., Myrianthopoulos, V., Magiatis, P., Grant, K. M., Meijer, L., Mikros, E., Skaltsounis, A. L. and Soteriadou, K. (2009). 6-Br-5methylindirubin-3'oxime (5-Me-6-BIO) targeting the leishmanial glycogen synthase kinase-3 (GSK-3) short form affects cell-cycle progression and induces apoptosis-like death: exploitation of GSK-3 for treating leishmaniasis. Int J Parasitol 39(12): 1289-1303. 